\title{
TIGHT NEIGHBORHOODS OF CONTACT SUBMANIFOLDS
}

\author{
LUIS HERNÁNDEZ-CORBATO, LUCÍA MARTÍN-MERCHÁN, AND FRANCISCO PRESAS
}

\begin{abstract}
We prove that any small enough neighborhood of a closed contact submanifold is always tight under a mild assumption on its normal bundle. The non-existence of $C^{0}$-small positive loops of contactomorphisms in general overtwisted manifolds is shown as a corollary.
\end{abstract}

\section{INTRODUCTION}

A contact manifold $(M, \xi)$ is an $(2 n+1)$-dimensional manifold equipped with a maximally non-integrable codimension 1 distribution $\xi \subset T M$. If we assume that $\xi$ is coorientable, as will be the case in the article, the hyperplane distribution can be written as the kernel of a global 1 -form $\alpha, \xi=\operatorname{ker}(\alpha)$, and the maximal non-integrable condition reads as $\alpha \wedge(d \alpha)^{n} \neq 0$. These conditions imply that $(\xi, d \alpha)$ is a symplectic vector bundle over $M$. However, a contact structure on $M$ cannot be directly recovered from a hyperplane distribution $\xi$ and a symplectic structure $\omega$ on the fibers. The formal data $(\xi, \omega)$ is called formal contact structure.

Let $\operatorname{Cont}(M)$ and $\mathfrak{F} \mathfrak{C o n t}(M)$ denote the set of contact and formal contact structures, respectively. Gromov proved that if $M$ is open the natural inclusion is a homotopy equivalence. The statement does not readily extend to closed manifolds. In dimension 3, Eliashberg introduced a subclass $\mathfrak{C o n t}_{O T}(M)$ of $\mathfrak{C o n t}(M)$, the so-called overtwisted contact structures, and proved that any formal contact homotopy class contains a unique, up to isotopy, overtwisted contact structure. Recently, this result has been extended to arbitrary dimension in [2] so the notion of overtwisted contact structure has been settled in general.

Prior to [2], different proposals for the definition of the overtwisting phenomenum appeared in the literature. The plastikstufe, introduced in [12], resembled the overtwisted disk in the sense that it provides an obstruction to symplectic fillability. The presence of a plastikstufe has been shown to be equivalent to the contact structure being overtwisted (check [3, Theorem 1.1] and [11] for a list of disguises of an overtwisted structure). One of the corollaries obtained in [3] is a stability property for overtwisted structures: if $(M, \operatorname{ker} \alpha)$ is overtwisted then $(M \times$ $\left.\mathbb{D}^{2}(R), \operatorname{ker}\left(\alpha+r^{2} d \theta\right)\right)$ is also overtwisted provided $R>0$ is large enough, where $\mathbb{D}^{2}(R)$ denotes the open 2 -disk of radius $R$ and $r^{2} d \theta$ denotes the standard radial Liouville form in $\mathbb{R}^{2}$.

1.1. Statements of the results. This paper explores the other end of the previous discussion, can small neighborhoods of contact submanifolds be overtwisted? We provide a negative answer to the question in several instances. The main result presented in the article is the following:

Theorem 1. Let $(M, \operatorname{ker} \alpha)$ be a contact manifold. Then there exists $\varepsilon>0$ such that $(M \times$ $\left.\mathbb{D}^{2}(\varepsilon), \operatorname{ker}\left(\alpha+r^{2} d \theta\right)\right)$ is tight.

This theorem was previously obtained by Gironella [9, Corollary H] in the case of 3-manifolds with a completely different approach. An interesting consequence is stated in the next corollary:

Corollary 2. Given any overtwisted contact manifold $(M, \alpha)$, there exists a radius $R_{0} \in \mathbb{R}^{+} \backslash\{0\}$ such that $\left(M \times \mathbb{D}^{2}(R), \alpha+r^{2} d \theta\right)$ is tight if $R \in\left(0, R_{0}\right)$ and is overtwisted if $R>R_{0}$.

Note that a similar statement was already proven in [13] but in the case of GPS-overtwisted.

Theorem 1 can be extended to arbitrary neighborhoods of codimension 2 contact submanifolds $M$ whose normal bundle has a nowhere vanishing section:

2010 Mathematics Subject Classification. Primary 37J10. Secondary: 37C40, 37J55.

Key words and phrases. Contactomorphism, overtwisted, orderable, Hamiltonian. 
Theorem 3. Suppose $M$ is a contact submanifold of the contact manifold $(N, \xi)$. Assume that the normal bundle of $M$ has a nowhere vanishing section. Then, there is a neighborhood of $M$ in $N$ that is tight.

The proof of Theorems 1 and 3 is based on Theorem 10. That theorem states that for $m$ large enough $\left(M \times P^{2 m}(\varepsilon), \operatorname{ker}\left(\alpha+\sum_{i=1}^{m} r_{i}^{2} d \theta_{i}\right)\right)$ admits a contact embedding in a closed contact manifold of the same dimension that is Stein fillable, therefore $M \times P^{2 m}(\varepsilon)$ is obviously tight. However, Theorems 1 and 3 do not prove such a strong result. Their proof uses [3, Theorem 1.1.(ii)] and some packing lemmas to obtain a contradiction by stabilizing and reducing to Theorem 10.

\subsection{Applications.}

1.2.1. Remarks about contact submanifolds. We are assuming a choice of contact forms whenever the measure of a radius of the tubular neighborhoods of a contact submanifold is required.

1. Assume that $(M, \xi)$ contact embeds into an overtwisted contact manifold $\left(N, \xi_{O T}\right)$ as a codimension 2 submanifold with trivial normal bundle. By Theorem 1, it is clear that the overtwisted disk cannot be localized on arbitrary small neighborhoods of $M$, even assuming that $M$ itself is overtwisted. This stands in sharp contrast with [11] and [3] in which it is shown that the overtwisted disk can be localized around a very special kind of codimension $n$ submanifold: a plastikstufe [12].

2. Assume now that $\left(M, \xi_{O T}\right)$ is overtwisted and contact embeds into a tight contact manifold $(N, \xi)$ as codimension 2 submanifold with trivial normal bundle. Then we can perform a fibered connected sum of $(N, \xi)$ with itself along $\left(M, \xi_{O T}\right)$. The gluing region is $M \times(-\varepsilon, \varepsilon) \times \mathbb{S}^{1}$, for some $\varepsilon>0$, and coordinates $(p, t, \theta)$ can be chosen such that the glued contact structure admits an associated contact form $\alpha=\alpha_{O T}+t d \theta$.

It is clear that the contact connection associated to the contact fibration $M \times(-\varepsilon, \varepsilon) \times \mathbb{S}^{1} \rightarrow$ $(-\varepsilon, \varepsilon) \times \mathbb{S}^{1}[14]$ induces the identity when we lift by parallel transport the loop $\{0\} \times \mathbb{S}^{1}$. The parallel transport of an overtwisted disk of the fiber induces a plastikstufe, see [14] for more details. By [11], the manifold is overtwisted.

Call $R_{M}>0$ the biggest radius for which $M \times \mathbb{D}^{2}\left(R_{M}\right)$ contact embeds in $N$. The connected sum $N \#_{M} N$ readily increases the biggest radius to be $R_{N \#_{M} N} \geq \sqrt{2} R_{M}$ : the annulus has twice the area of the original disk and therefore you can embed a disk of radius $\sqrt{2} R_{M}$. However we get much more, since we actually obtain $R_{N{ }_{M} N}=\infty$. This is because we can always formally contact embed $M \times \mathbb{R}^{2}$ into $N \#_{M} N$. Moreover, we can assume that the embedding restricted to a very small neighborhood $U$ of the fiber $M \times\{0\}$ provides a honest fibered contact embedding into $M \times(-\varepsilon, \varepsilon) \times \mathbb{S}^{1}$. Indeed, applying [2, Corollary 1.4] relative to the domain $U$ we obtain a contact embedding of $M \times \mathbb{R}^{2}$ thanks to the fact that $N \#_{M} N$ is overtwisted. This just means that the contact embedding of the tubular neighborhood can be really sophisticated and its explicit construction is far from obvious.

1.2.2. Small loops of contactomorphisms. Theorem 1 allows to extend the result of non-existence of small positive loops of contactomorphisms in overtwisted 3-manifolds contained in [4] to arbitrary dimension. A loop of contactomorphisms or, more generally, a contact isotopy is said to be positive if it moves every point in a direction positively transverse to the contact distribution. The notion of positivity induces for certain manifolds, called orderable, a partial order on the universal cover of the contactomorphism group and it is related with non-squeezing and rigidity in contact geometry, see [6, 8]. As explained in [6], orderability is equivalent to the non-existence of a positive contractible loop of contactomorphisms.

Any contact isotopy is generated by a contact Hamiltonian $H_{t}: M \rightarrow \mathbb{R}$ that takes only positive values in case the isotopy is positive. The main result of [4] states that if $(M, \operatorname{ker} \alpha)$ is an overtwisted 3-manifold there exists a constant $C(\alpha)$ such that any positive loop of contactomorphisms generated by a Hamiltonian $H: M \times S^{1} \rightarrow \mathbb{R}^{+}$satisfies $\|H\|_{\mathcal{C}^{0}} \geq C(\alpha)$. The result has been recently extended to arbitrary hypertight or Liouville (exact symplectically) fillable 
contact manifolds in [1]. As a consequence of Theorem 1, we can eliminate the restriction on the dimension in the overtwisted case:

Theorem 4. Let $(M, \operatorname{ker} \alpha)$ be an overtwisted contact manifold. There exists a constant $C(\alpha)$ such that the norm of a Hamiltonian $H: M \times S^{1} \rightarrow \mathbb{R}^{+}$that generates a positive loop $\left\{\phi_{\theta}\right\}$ of contactomorphisms on $M$ satisfies

$$
\|H\|_{\mathcal{C}^{0}} \geq C(\alpha)
$$

The strategy of the proof copies that of [4]. The first step is to prove that $M \times \mathbb{D}^{2}(\varepsilon)$ is tight, this is provided by Theorem 1 . The second step shows that a small positive loop provides a way to lift a plastikstufe in $M$ (whose existence is equivalent to overtwistedness as discussed above [11]) to a plastikstufe in $M \times \mathbb{D}^{2}(\varepsilon)$. This is exactly Proposition 9 in [4]. This provides a contradiction that forbids the existence of the small positive loop.

It is worth mentioning that the argument forbids the existence of (possibly non-contractible) small positive loops. This is in contrast with [1] and the work in progress by S. Sandon [15] in which they need to add the contractibility hypothesis in order to conclude.

Remark 5. The hypothesis in Theorem 4 can be changed by the probably weaker notion of $G P S$-overtwisted, see [13]. Indeed, assume that the manifold $(M, \xi)$ is GPS-overtwisted. This means that there is an immersed GPS in the manifold. The positive loop produce a GPS in $M \times \mathbb{D}^{2}(\varepsilon)$ by parallel transport of the $G P S$ around a closed loop in the base $D^{2}(\varepsilon)$. In this case, we need to iterate the process $k$ times to produce a GPS in $M \times P^{2 k}(\varepsilon)$. Now, Theorem 10 concludes that this manifold embeds into a Stein fillable one providing a contradiction with the main result in [13].

\section{ACKNOWLEDGEMENTS}

The authors express their gratitude to Roger Casals for the useful conversations around this article. The authors have been supported by the Spanish Research Projects SEV-20150554, MTM2015-63612-P, MTM2015-72876-EXP and MTM2016-79400-P. The second author was supported during the development of the article by a Master grant from ICMAT through the Severo Ochoa program.

\section{2. $M \times P^{2 m}(\varepsilon)$ ADMits A Stein Fillable SMOOTH COMPACTIFICATION}

2.1. Construction of a formal contact embedding $M \rightarrow \partial W$ with trivial normal bundle. Recall that $(\xi, d \alpha)$ defines a symplectic vector bundle over $M$, thus it is equipped with a complex bundle structure unique up to homotopy. Denote $\xi^{*}$ the dual complex vector bundle of $\xi$. A standard result on the theory of vector bundles guarantees the existence of a complex vector bundle $\tau \rightarrow M$ such that $\xi^{*} \oplus \tau \rightarrow M$ is trivial, that is, there is an isomorphism of complex vector bundles over $M$ between $\xi^{*} \oplus \tau$ and $M \times \mathbb{C}^{k}=\underline{\mathbb{C}}^{k}$, where $k$ is a positive integer large enough.

Denote $\pi: T^{*} M \rightarrow M$ the cotangent bundle projection and denote pr: $\pi^{*} \tau \rightarrow T^{*} M$ the bundle projection. Define $\tilde{\pi}=\pi \circ$ pr. Let us understand $\widehat{W}=\pi^{*} \tau$ as a smooth almost complex manifold. Choosing a $\xi$-compatible contact form $\alpha$, i.e $\xi=\operatorname{ker} \alpha$, it is clear that

$$
\begin{aligned}
T \widehat{W} & \cong \tilde{\pi}^{*} \tau \oplus \operatorname{pr}^{*} T\left(T^{*} M\right) \cong \tilde{\pi}^{*} \tau \oplus \tilde{\pi}^{*} T^{*} M \oplus \tilde{\pi}^{*} T M \cong \tilde{\pi}^{*} \tau \oplus \tilde{\pi}^{*}\left(\xi^{*} \oplus\langle\alpha\rangle\right) \oplus \tilde{\pi}^{*} T M \\
& \cong \tilde{\pi}^{*}\left(\tau \oplus \xi^{*}\right) \oplus \tilde{\pi}^{*}\langle\alpha\rangle \oplus \tilde{\pi}^{*} T M \cong \tilde{\pi}^{*} \underline{\mathbb{C}}^{k} \oplus \tilde{\pi}^{*}\langle\alpha\rangle \oplus \tilde{\pi}^{*} T M
\end{aligned}
$$

In particular, the vector bundle $\pi^{*} \tau \stackrel{\tilde{\pi}}{\rightarrow} M$ is isomorphic to $\underline{\mathbb{C}}^{k} \oplus\langle\alpha\rangle$. Fix a direct sum bundle metric $h$ in $\pi^{*} \tau$ such that $h(\alpha, \alpha)=1$. Now define

$$
W=\{(v, p) \in \widehat{W}: h(v, v) \leq 1\} .
$$

Given a complex structure $j$ in $\xi$ compatible with $d \alpha$, we can extend it to a complex structure on $T^{*} M$ and by a direct sum with a complex structure in $\tau$ we obtain a complex structure $J$ 
in $T \widehat{W}$. Then, $(W, J)$ is an almost complex manifold with boundary $\partial W$ that has a natural formal contact structure $\xi_{0}=T \partial W \cap J(T \partial W)$. Consider the embedding

$$
e_{0}: M \rightarrow \partial W=\mathbb{S}\left(\underline{\mathbb{C}}^{k} \oplus\langle\alpha\rangle\right): p \mapsto(0,1) .
$$

We claim that its normal bundle is trivial because it is equal to $\tilde{\pi}^{*} \mathbb{C}^{k}$. The reason is that the normal bundle to a section of a vector bundle is the restriction of the vertical bundle to the section. In our case the restriction of the vertical bundle $\left.T\left(\mathbb{S}\left(\underline{\mathbb{C}}^{k}\right) \oplus\langle\alpha\rangle\right)\right)$ to the image of $e_{0}$ is clearly $\left(\tilde{\pi}^{*} \underline{\mathbb{C}}^{k}\right)_{\mid i m\left(e_{0}\right)}$.

2.2. $W$ is Stein fillable and $\partial W$ is contact. The distribution $T \partial W \cap J(T \partial W)$ is not necessarily a contact structure in $\partial W$. However, we will deform this distribution to a genuine contact structure using the following result.

Theorem 6 (Eliashberg [7]). Let $\left(V^{2 n}, J\right)$ be an almost complex manifold with boundary of dimension $2 n>4$ and suppose that $f: V \rightarrow[0,1]$ is a Morse function constant on $\partial V$ such that $\operatorname{ind}_{p}(f) \leq n$ for every $p \in \operatorname{Crit}(f)$. Then, there exists a homotopy of almost complex structures $\left\{J_{t}\right\}_{t=0}^{1}$ such that $J_{0}=J, J_{1}$ is integrable and $f$ is $J_{1}$-convex.

We are clearly in the hypothesis since our manifold $W$ is almost complex, has dimension $2 k+1+\operatorname{dim} M>4$ (because $2 k \geq \operatorname{dim} \xi=\operatorname{dim} M-1$ ) and deformation retracts to $M$.

From Theorem 6 we obtain a homotopy of almost complex structures $\left\{J_{t}\right\}$ in $W$ such that $J_{0}=J$ and, $J_{1}$ is integrable. Moreover $\left(W, J_{1}\right)$ is a Stein domain and $\partial W$ inherits a contact structure given by $\xi_{1}=J_{1}(T \partial W) \cap T \partial W$. In fact, there is a homotopy of formal contact structures between $\xi_{0}$ and $\xi_{1}$ provided by $\xi_{t}=J_{t}(T \partial W) \cap T \partial W$.

2.3. Properties of the embedding $e_{0}:(M, \xi) \rightarrow\left(\partial W, \xi_{1}\right)$. Recall the following definition:

Definition 7. An embedding $e:\left(M_{0}, \xi_{0}, J_{0}\right) \rightarrow\left(M_{1}, \xi_{1}, J_{1}\right)$ is called formal contact if there exists an homotopy of monomorphisms $\left\{\Psi_{t}: T M_{0} \rightarrow T M_{1}\right\}_{t=0}^{1}$ such that $\Psi_{0}=d e, \xi_{0}=\Psi_{1}^{-1}\left(\xi_{1}\right)$ and $\Psi_{1}:\left(\xi_{0}, J_{0}\right) \rightarrow\left(\xi_{1}, J_{1}\right)$ is complex.

So far we have produced an embedding $e_{0}:(M, \xi, j) \rightarrow\left(\partial W, \xi_{0}, J_{0}\right)$ that is formal contact with the constant homotopy equal to $d e_{0}$. Indeed, $d e_{0}^{-1}\left(\xi_{0}\right)=\xi$ and $d e_{0}(\xi)$ is a complex subbundle of $\xi_{0}$. There is a family of complex isomorphisms $\Phi_{t}: \xi_{0} \rightarrow \xi_{t}$ such that $\Phi_{0}=$ id. Fix a Reeb vector field $R$ associated to $\xi$ and define $\widehat{R}_{0}=d e_{0}(R)$. Build a family $\left\{R_{t}\right\}$ of vector fields in $T \partial W$ satisfying $\left.R_{0}\right|_{\text {im } e_{0}}=\widehat{R}_{0}$ and $\left\langle R_{t}\right\rangle \oplus \xi_{t}=T \partial W$. We take a family of metrics $g_{t}$ in $\partial W$ defined in the following way: its restriction to $\xi_{t}$ is hermitic for the complex bundle $\left(\xi_{t}, J_{t}\right)$ and $R_{t}$ is unitary and orthogonal to $\xi_{t}$.

Extend $\Phi_{t}$ to an isomorphism of $\left.T \partial W\right|_{\mathrm{im} e_{0}}$ in such a way that $\Phi_{t}\left(R_{0}\right)=R_{t}$. Define

$$
E_{t}=\Phi_{t} \circ d e_{0}: T M \rightarrow T \partial W .
$$

The family $\left\{E_{t}\right\}_{t=0}^{1}$ is composed of bundle monomorphisms and clearly satisfies that $E_{1}^{-1}\left(\xi_{t}\right)=\xi$ and $E_{1}(\xi)$ is a complex subbundle of $\xi_{1}$. Therefore, $\left(e_{0}, E_{t}\right)$ is a formal contact embedding.

Define $\mathcal{N}_{t}=E_{t}(T M)^{\perp g_{t}}$ that is a bundle over im $e_{0}$ which is complex by construction. $\mathcal{N}_{0}$ is isomorphic to $\underline{\mathbb{C}}^{k}$ and therefore all the bundles $\mathcal{N}_{t}$ are trivial complex bundles.

2.4. Obtaining a contact embedding via $\mathbf{h}-$ principle. The only missing piece to complete the puzzle is to prove that the embedding $e_{0}$ can be made contact.

Using $h$-principle it is possible to deform $\left(e_{0}, E_{t}\right)$ to a contact embedding thanks to the following theorem (cf. [5, Theorem 12.3.1]):

Theorem 8. Let $\left(e, E_{t}\right)$, e: $\left(M_{0}, \xi_{0}=\operatorname{ker} \alpha_{0}\right) \rightarrow\left(M_{1}, \xi_{1}=\operatorname{ker} \alpha_{1}\right)$, be a formal contact embedding between closed contact manifolds such that $\operatorname{dim} M_{0}+2<\operatorname{dim} M_{1}$. Then, there exists a family of embeddings $\widetilde{e}_{t}: M_{0} \rightarrow M_{1}$ such that:

- $\widetilde{e}_{0}=e$ and $\widetilde{e}_{1}$ is contact,

- d $\widetilde{e}_{1}$ is homotopic to $E_{1}$ through monomorphisms $G_{t}: T M_{0} \rightarrow T M_{1}$, lifting the embeddings $\widetilde{e}_{t}$, such that $G_{t}\left(\xi_{0}\right) \subset \xi_{1}$ and the restrictions $\left.G_{t}\right|_{\xi_{0}}:\left(\xi_{0}, d \alpha_{0}\right) \rightarrow\left(\xi_{1}, d \alpha_{1}\right)$ are symplectic. 
Theorem 8 applied to $\left(e_{0}, E_{t}\right)$ provides a family of embeddings $\left\{e_{t}\right\}$ in which $e_{1}:(M, \xi) \rightarrow$ $\left(\partial W, \xi_{1}\right)$ is a contact embedding and a family of monomorphisms $G_{t}: T M \rightarrow T \partial W$ that lift $e_{t}$ such that $G_{0}=E_{1}, G_{1}=d e_{1}$ and $G_{t}(\xi) \subset \xi_{1}$ is a complex subbundle.

Lemma 9. The normal bundle of $\operatorname{im}\left(e_{1}\right)$ in $\left(\partial W, \xi_{1}\right)$ is trivial.

Proof. Recall that $\mathcal{N}_{1}=E_{1}(T M)^{\perp g_{1}}=G_{0}(T M)^{\perp g_{1}}$ is a trivial complex vector bundle. Define, for $t \in[1,2], \mathcal{N}_{t}=G_{t-1}(T M)^{\perp g_{1}}$. Clearly, $\mathcal{N}_{2}$ is the normal bundle of the contact embedding $e_{1}$. Since $\mathcal{N}_{1}$ is a trivial vector bundle so is $\mathcal{N}_{2}$.

Denote the $2 m$-dimensional polydisk by $P^{2 m}\left(r_{1}, \ldots, r_{m}\right)=\mathbb{D}^{2}\left(r_{1}\right) \times \cdots \times \mathbb{D}^{2}\left(r_{m}\right)$ and abbreviate it as $P^{2 m}(r)$ when $r_{1}=\ldots=r_{m}=r$. The following result summarizes the work completed in this section and an important consequence (namely, the title of the section): $M \times P^{2 m}(\varepsilon)$ admits a smooth compactification into a Stein fillable contact manifold.

Theorem 10. Any closed contact manifold $(M, \operatorname{ker} \alpha)$ contact embeds in the boundary of a Stein fillable manifold with trivial normal bundle. Furthermore, there exists $k \geq 1$ such that for any $m \geq k$

$$
\left(M \times P^{2 m}(\varepsilon), \operatorname{ker}\left(\alpha+\sum_{i=1}^{m} r_{i}^{2} d \theta_{i}\right)\right)
$$

is tight with $\varepsilon>0$ small enough depending only on $\alpha$ and $k$.

Proof. The map $e_{1}$ proves the first part because by Lemma 9 the normal bundle of the contact embedding $e_{1}:(M, \xi) \rightarrow\left(\partial W, \xi_{1}\right)$ is trivial. Notice that the codimension of the embedding is equal to $2 k=\operatorname{dim} \tau$ and by replacing $\tau$ with $\tau^{\prime}=\tau \oplus \underline{\mathbb{C}}^{m-k}$ we obtain embeddings of arbitrary codimension $2 m \geq 2 k$.

Suppose henceforth that $m \geq k$. By an standard neighborhood theorem in contact geometry it follows that there is a contactomorphism between a neighborhood of $\operatorname{im}\left(e_{1}\right)$ in $\left(\partial W, \xi_{1}\right)$ and a neighborhood of $M \times\{0\}$ in $\left(M \times \mathbb{R}^{2 m}, \operatorname{ker}\left(\alpha+\sum_{i=1}^{k} r_{i}^{2} d \theta_{i}\right)\right)$. Therefore, for some $\varepsilon_{0}>0$, the previous contactomorphism provides an embedding from $M \times P^{2 m}\left(\varepsilon_{0}\right)$ into $\partial W$.

Finally, since $\left(\partial W, \xi_{1}\right)$ is Stein filable, it is tight. Thus, any of its open subsets is also tight and the conclusion follows.

\section{3. $M \times \mathbb{D}^{2}(\varepsilon)$ IS TIGHT IF $\varepsilon$ IS SMALL}

The argument leading to Theorem 10 provided no bound on the first positive integer $k$ such that $M \times P^{2 k}(\varepsilon, \ldots, \varepsilon)$ is tight. Indeed, $k$ was fixed at the beginning of Section 2 , depending on the rank of $\tau \rightarrow M$, the bundle constructed to make the sum $\xi^{*} \oplus \tau$ trivial.

The insight needed to prove Theorem 1 is supplied by the understanding of overtwisted contact manifolds briefly discussed in the introduction. To be more concrete, the precise statement we will use in this section, extracted from [3], is the following:

Theorem 11. Suppose that $(M, \operatorname{ker} \alpha)$ is an overtwisted contact manifold. Then, if $R$ is large enough, $\left(M \times \mathbb{D}^{2}(R), \operatorname{ker}\left(\alpha+r^{2} d \theta\right)\right)$ is also overtwisted.

The idea is to embed $\partial W \times \mathbb{D}^{2}(R)$ in the boundary $\partial V$ of a Weinstein manifold. Using the embedding constructed in the previous section we obtain then an embedding $M \times \mathbb{D}^{2}(R) \rightarrow \partial V$ that has trivial normal bundle. This leads to the proof of a statement similar to Theorem 10 in which we replace $(M, \operatorname{ker} \alpha)$ by $\left(M \times \mathbb{D}^{2}(R), \operatorname{ker}\left(\alpha+r^{2} d \theta\right)\right)$. Note that it is key to make sure that $R$ is arbitrarily large.

A Weinstein manifold $(W, \omega, f, Y)$ is a manifold with boundary $W$ equipped with a symplectic structure $\omega$, a Morse function $f: W \rightarrow \mathbb{R}$ and a Liouville vector field $Y$ that is a pseudogradient for $f$. Notice that the symplectic form is automatically exact, $\omega=L_{Y} \omega=d i_{Y} \omega$, so the boundary of a Weinstein manifold is exact symplectically fillable.

The product of Weinstein manifolds $\left(W_{1}, \omega_{1}, g_{1}, Y_{1}\right)$ and $\left(W_{2}, \omega_{2}, g_{2}, Y_{2}\right)$ can be equipped with a Weinstein structure. Indeed, define $\omega^{\prime}=\omega_{1}+\omega_{2}$ and $Y^{\prime}=Y_{1}+Y_{2}$. Clearly, $Y^{\prime}$ is Liouville 
for $\omega^{\prime}$. Suppose for simplicity that $g_{1}$ and $g_{2}$ are strictly positive (a rescaling would make the argument work in general) and define a function on $W=W_{1} \times W_{2}$ by

$$
f_{q}=\left(g_{1}^{q}+g_{2}^{q}\right)^{1 / q}
$$

for an arbitrary $q>1$. It is easy to check that $\operatorname{Crit}\left(f_{q}\right)=\operatorname{Crit}\left(g_{1}\right) \times \operatorname{Crit}\left(g_{2}\right)$, the function $f_{q}$ is Morse and $Y^{\prime}$ is pseudogradient for $f_{q}$.

The Stein fillable manifold $W$ supplied by Theorem 10 is naturally equipped with a Weinstein structure $\left(W=f^{-1}(0,1], \omega, f, Y\right)$ that satisfies $\xi_{1}=\operatorname{ker}\left(\left.i_{Y} \omega\right|_{\partial W}\right)$. By the preceeding discussion, a Weinstein structure in $W \times \mathbb{R}^{2}$ is given by $\omega+d x \wedge d y, X=Y+r \frac{\partial}{\partial r}$ and

$$
f_{q}=\left(f^{q}+\left(\frac{x^{2}+y^{2}}{(2 R)^{2}}\right)^{q}\right)^{1 / q}
$$

The critical points of $f_{q}$ have the form $(p, 0,0)$, where $p \in \operatorname{Crit}(f)$.

The Liouville vector field $X$ is transverse to $\partial W \times \mathbb{R}^{2}$. Our aim is now to embed $\partial W \times \mathbb{D}^{2}(R)$ into a level set of $f_{q}$ by following $\phi_{t}$, the flow of $X$. We can easily show:

Proposition 12. For any $\delta>0$, there exists $q>1$ large enough and a function $\mu: \partial W \times$ $\mathbb{D}^{2}(R) \rightarrow \mathbb{R}^{-}$such that $\|\mu\|_{\mathcal{C}^{0}} \leq \delta$ and $\phi_{\mu}: \partial W \times \mathbb{D}^{2}(R) \rightarrow W \times \mathbb{D}^{2}(R)$ satisfies $\phi_{\mu}(\partial W \times$ $\left.\mathbb{D}^{2}(R)\right) \subset f_{q}^{-1}(1)$.

Proof. For $q \rightarrow \infty$, the level set $f_{q}^{-1}(1)$ gets $C^{\infty}$-close to the submanifold $\partial W \times \mathbb{D}^{2}(R)$. Since $X$ is transverse to both of them, the result follows.

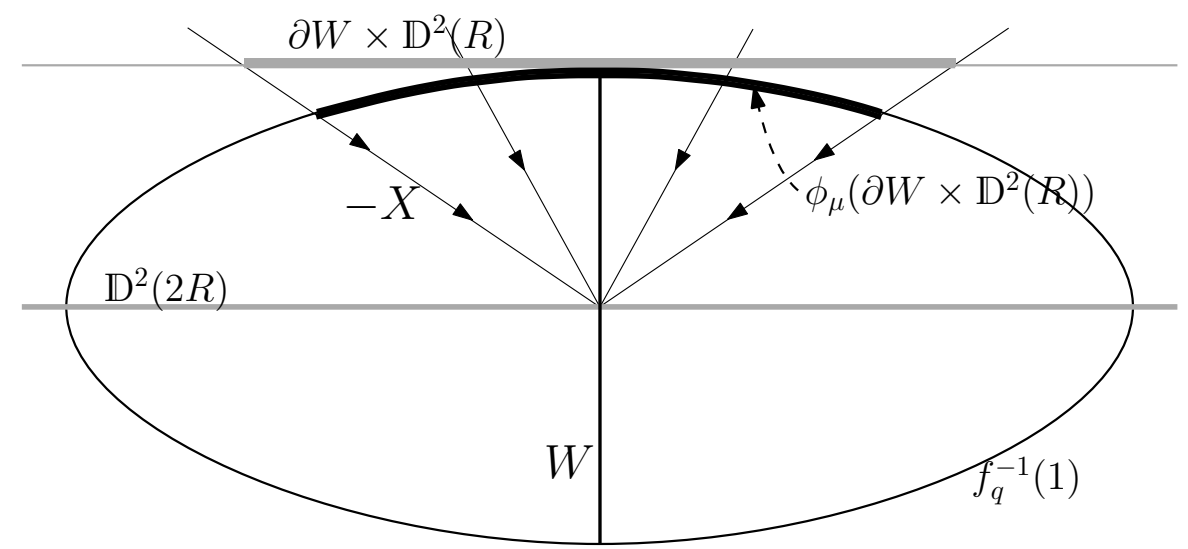

Figure 1. Contact embedding of $\partial W \times \mathbb{D}^{2}(R)$ into $f_{q}^{-1}(1)$.

Proposition 12 produces a contactomorphism as the next lemma states.

Lemma 13. Let $e: H \hookrightarrow M$ be a hypersurface transverse to a nowhere vanishing Liouville vector field $X$ in $(M, \omega)$, the 1 -form $e^{*} i_{X} \omega$ defines a contact structure on $H$. Moreover, if $\phi_{t}$ denotes the Liouville flow starting at $H$ and $s: H \rightarrow \mathbb{R}$ is a fixed function, then $\phi_{s} \circ e: H \hookrightarrow M$ is contactomorphic to e provided the flow $\phi_{s}$ is well-defined.

Notice that the level set $f_{q}^{-1}(1)$ is the boundary of the Weinstein manifold $V=f_{q}^{-1}(0,1]$. Denote $\alpha^{\prime}=i_{X}(\alpha+d x \wedge d y)$. A straightforward application of Lemma 13 concludes the following:

Proposition 14. For any $R>0$, the contact manifold $\left(\partial W \times \mathbb{D}^{2}(R), \operatorname{ker}\left(\left.\alpha^{\prime}\right|_{\partial W \times \mathbb{D}^{2}(R)}\right)\right)$ admits a contact embedding into the boundary of a Weinstein manifold.

Combining the last proposition and the results from the previous section we obtain:

Corollary 15. Given a contact manifold $(M, \alpha)$ there exists $k \in \mathbb{N}$ and $\varepsilon_{0}>0$ such that for every $R>0$ the contact manifold $\left(M \times P^{2 k+2}\left(\varepsilon_{0}, \ldots, \varepsilon_{0}, R\right), \operatorname{ker}\left(\alpha+\sum_{i=1}^{k+1} r_{i}^{2} d \theta_{i}\right)\right)$ is tight. 
Let us emphasize that $\varepsilon_{0}$ does not depend on $R$ : for any $R>0, M \times P^{2 k+2}\left(\varepsilon_{0}, \ldots, \varepsilon_{0}, R\right)$ is tight.

Proof. The integer $k$ and the number $\varepsilon_{0}$ both come from Theorem 10. Denote by $e^{\prime}$ the contact embedding from $\left(M \times P^{2 k}\left(\varepsilon_{0}\right), \operatorname{ker}\left(\alpha+\sum_{i=1}^{k} r_{i}^{2} d \theta_{i}\right)\right)$ into $\left(\partial W, \xi_{1}=\operatorname{ker}\left(i_{Y} \omega\right)\right)$ and let $\eta$ be the conformal factor of $e^{\prime},\left(e^{\prime}\right)^{*} i_{Y} \omega=\exp (\eta) \alpha^{\prime}$. If necessary, decrease the value of $\varepsilon_{0}$ to guarantee that $\sup \eta$ is finite.

Proposition 14 supplies a Weinstein manifold $\left(V=f_{q}^{-1}(0,1], \omega+d x \wedge d y, f_{q}, X\right)$ and contact embedding

$$
\varphi:\left(\partial W \times \mathbb{D}^{2}(\exp (\sup \eta / 2) R), \alpha^{\prime}\right) \hookrightarrow\left(\partial V, \alpha^{\prime}\right) .
$$

Therefore, the map $\widetilde{\varphi}: M \times P^{2 k+2}\left(\varepsilon_{0}, \ldots, \varepsilon_{0}, R\right) \rightarrow \partial V$ given by

$$
\left.\widetilde{\varphi}\left(p, x, y, x_{k+1}, y_{k+1}\right)=\varphi\left(e^{\prime}(p, x, y), \exp (\eta / 2) x_{k+1}, \exp (\eta / 2) y_{k+1}\right)\right)
$$

is a contact embedding. Since $\partial V$ is exact symplectically fillable the conclusion follows.

We are ready now to prove Theorem 1 . To ease the notation, we shall understand the contact form is equal to $\alpha+\sum r_{i}^{2} d \theta_{i}$ in case it is omitted.

Let us proceed by contradiction. Suppose that $M \times \mathbb{D}^{2}(\varepsilon)$ is overtwisted for $\varepsilon$ smaller than $\varepsilon_{0}$. Applying Theorem $11 k$ times consecutively we obtain a radius $R_{\varepsilon}>0$ such that $M \times$ $P^{2 k+2}\left(\varepsilon, R_{\varepsilon}, \ldots, R_{\varepsilon}\right)$ is overtwisted. As we will show below, this manifold contact embeds into $M \times P^{2 k+2}\left(\varepsilon_{0}, \ldots, \varepsilon_{0}, R\right)$ provided $R$ is large enough. From Corollary 15 we know that the latter manifold is tight so we reach a contradiction. Therefore, $M \times \mathbb{D}^{2}(\varepsilon)$ is tight.

The only missing ingredient is the announced contact embedding:

$$
M \times P^{2 k+2}\left(\varepsilon, R_{\varepsilon}, \ldots, R_{\varepsilon}\right) \rightarrow M \times P^{2 k+2}\left(\varepsilon_{0}, \ldots, \varepsilon_{0}, R\right)
$$

Its existence, subject to the conditions $\varepsilon<\varepsilon_{0}$ and $R$ large enough, is a consequence of the following packing theorem in symplectic geometry proved by Guth [10, Theorem 1].

Theorem 16. For every $m \in \mathbb{N}$ there is a constant $C(m) \geq 1$ such that for any pair of ordered $m$-tuples of positive numbers $R_{1} \leq \ldots \leq R_{m}$ and $R_{1}^{\prime} \leq \ldots \leq R_{m}^{\prime}$ that satisfy

- $C(m) R_{1} \leq R_{1}^{\prime}$ and

$$
\text { - } C(m) R_{1} \cdot \ldots \cdot R_{k} \leq R_{1}^{\prime} \cdot \ldots \cdot R_{m}^{\prime} \text {. }
$$

there is a symplectic embedding

$$
P^{2 m}\left(R_{1}, \ldots, R_{m}\right) \hookrightarrow P^{2 m}\left(R_{1}^{\prime}, \ldots, R_{m}^{\prime}\right)
$$

The symplectic embedding supplied by Theorem 16 is automatically extended to our desired contact embedding (1) thanks to the following lemma:

Lemma 17. Let $\Psi:\left(D_{1}, d \lambda_{1}\right) \rightarrow\left(D_{2}, d \lambda_{2}\right)$ be an exact symplectic embedding. For any contact manifold $(M, \operatorname{ker} \alpha)$ with a choice of contact form $\alpha$ that makes the associated Reeb flow complete, $\Psi$ induces a (strict) contact embedding

$$
\left(M \times D_{1}, \alpha+\lambda_{1}\right) \rightarrow\left(M \times D_{2}, \alpha+\lambda_{2}\right) .
$$

Proof. Since $\Psi$ is exact, there exists a smooth function $H: D_{1} \rightarrow \mathbb{R}$ such that $d H=\Psi^{*} \lambda_{2}-\lambda_{1}$. If we denote the Reeb flow in $M$ by $\Phi$,

$$
\varphi:\left(M \times D_{1}, \alpha+\lambda_{1}\right) \rightarrow\left(M \times D_{2}, \alpha+\lambda_{2}\right), \quad \varphi(p, x)=\left(\Phi_{-H(x)}(p), \Psi(x)\right)
$$

is a contact embedding. 


\section{EXtension to CONTACT SUBMANifolds}

The results from the previous sections can be extended to a more general setting: contact submanifolds with arbitrary normal bundle. In the presence of a nowhere vanishing section of the normal bundle we will prove that the contact submanifold has a tight neighborhood. This is the content of Theorem 3 .

Let $\pi: E \rightarrow M$ be a complex vector bundle over a contact manifold equipped with an hermitian metric and a unitary connection $\nabla$. The associated vertical bundle is denoted by $\mathcal{V}=\operatorname{ker}(d \pi)$. The standard Liouville form in $\mathbb{R}^{2 n}$ is $U(n)$-invariant and induces a global 1form in $\mathcal{V}$ that will be denoted $\widetilde{\lambda}$. This real 1 -form can be extended to $T E$ by the expression $\lambda=\tilde{\lambda}_{\circ} \circ \pi_{\mathcal{V}}$ after we choose a projection onto the vertical direction $\pi_{\mathcal{V}}: T E \rightarrow \mathcal{V}$. The map $\pi_{V}$ is determined by the choice of unitary connection so it is not canonical. The $1-$ form in $T E$ associated to the connection $\nabla$ is $\widetilde{\alpha}=\pi^{*} \alpha+\lambda$.

Even though $\widetilde{\alpha}$ can be seen as the lift of the contact form $\alpha$ to $E$, it is not a globally defined contact form in general. However, it defines a contact form around the zero section $E_{0}$ of the vector bundle.

Lemma 18. $\widetilde{\alpha}$ is a contact form in a neighborhood of $E_{0}$. The restriction $\left(E_{0}, \operatorname{ker}\left(\left.\widetilde{\alpha}\right|_{E_{0}}\right)\right)$ is contactomorphic to $(M, \xi=\operatorname{ker} \alpha)$. Moreover, given any other contact structure $\operatorname{ker} \beta$ that coincides with $\operatorname{ker}(\widetilde{\alpha})$ in $E_{0}$ and with the same complex structure in the normal bundle, there exist neighborhoods $U, V$ of $E_{0}$ such that $\left(U, \operatorname{ker}\left(\left.\beta\right|_{U}\right)\right)$ and $\left(V, \operatorname{ker}\left(\left.\widetilde{\alpha}\right|_{V}\right)\right)$ are contactomorphic.

Suppose henceforth that $\pi$ has a global nowhere vanishing section $s: M \rightarrow E$. The section $s$ creates a complex line subbundle $\left.\pi\right|_{L}: L \rightarrow M$. Then, the bundle $E$ splits as $E=F \oplus L$ and $L$ is trivial, i.e. there is an isomorphism $\phi: L \rightarrow \underline{\mathbb{C}}$ that sends $s(p)$ to $1_{p} \in \mathbb{C}$ in the fiber above every point $p \in M$.

A suitable choice of unitary connection on $\pi: E \rightarrow M$ ensures that the associated contact form can be written as $\widetilde{\alpha}=\alpha^{\prime}+\lambda$, where $\alpha^{\prime}$ is a contact form in $F$ and $\lambda$ is the radial Liouville form in $\mathbb{R}^{2}$.

Proposition 19. There exists $U$, a neighborhood of the zero section $F_{0}$ of $F$, and $\varepsilon>0$ such that $\left(U \times \mathbb{D}^{2}(\varepsilon), \operatorname{ker}\left(\alpha^{\prime}+\lambda\right)\right)$ is tight.

Note that this statement is exactly Theorem 1 except from the fact that $F$ is not closed. The proof of Proposition 19 follows by embedding $\left(U, \operatorname{ker} \alpha^{\prime}\right)$ in a closed contact manifold $\left(\widetilde{F}\right.$, ker $\left.\widetilde{\alpha}^{\prime}\right)$ and then applying Theorem 1 to this manifold to deduce that $\left(\widetilde{F} \times \mathbb{D}^{2}(\varepsilon), \operatorname{ker}\left(\widetilde{\alpha}^{\prime}+\lambda\right)\right)$ is tight if $\varepsilon>0$ is small. This result evidently implies that $\left(U \times \mathbb{D}^{2}(\varepsilon), \operatorname{ker}\left(\alpha^{\prime}+\lambda\right)\right)$ is also tight.

The aforementioned embedding is defined by the natural inclusion of $F$ in the projectivization of $F \oplus \mathbb{C}$ :

$$
F \hookrightarrow Q=\mathbb{P}(F \oplus \mathbb{C})
$$

The complex bundle $\pi_{Q}: Q \rightarrow M$ carries a natural formal contact structure $\xi^{\prime}=\left(d \pi_{Q}\right)^{-1}(\xi)$ Indeed, an almost complex structure in $\xi^{\prime}$ is obtained as the sum of the pullback of a complex structure in $\xi$ compatible with $d \alpha$ and a complex structure on the fibers of $\pi_{Q}$. This formal contact structure is genuine (i.e., it is a true contact structure) in a neighborhood $U$ of $F_{0}$ by Lemma 18. The $h$-principle for closed manifolds proved in [2, Theorem 1.1] provides a homotopy from any formal contact structure to a contact structure. Furthermore, the homotopy can be made relative to a closed set in which the formal contact structure is already genuine. Applying this theorem we obtain a contact structure $\widetilde{\xi}^{\prime}$ on $Q$ that agrees with $\operatorname{ker} \alpha^{\prime}$ in $U$.

We can reformulate Proposition 19 in the following way:

Theorem 20. Let $\pi: E \rightarrow M$ be a complex vector bundle over a closed contact manifold $(M, \xi)$. Suppose that $\pi$ has a global nowhere vanishing section. Then, there exists a neighborhood $U$ of the zero section of the bundle such that $(U, \widetilde{\xi})$ is tight for any contact structure $\widetilde{\xi}$ extending $\xi$ and preserving the complex structure of $E$.

An immediate application of Theorem 20 to the case in which $M$ is a contact submanifold and $\pi$ is its normal bundle yields Theorem 3 . 


\section{REFERENCES}

[1] Peter Albers, Urs Fuchs, and Will J. Merry. Positive loops and $L^{\infty}$-contact systolic inequalities. Selecta Math. (N.S.), 23(4):2491-2521, 2017.

[2] Matthew Strom Borman, Yakov Eliashberg, and Emmy Murphy. Existence and classification of overtwisted contact structures in all dimensions. Acta Math., 215(2):281-361, 2015.

[3] R. Casals, E. Murphy, and F. Presas. Geometric criteria for overtwistedness. ArXiv e-prints, March 2015.

[4] Roger Casals, Francisco Presas, and Sheila Sandon. Small positive loops on overtwisted manifolds. J. Symplectic Geom., 14(4):1013-1031, 2016.

[5] Y. Eliashberg and N. Mishachev. Introduction to the h-principle, volume 48 of Graduate Studies in Mathematics. American Mathematical Society, Providence, RI, 2002.

[6] Y. Eliashberg and L. Polterovich. Partially ordered groups and geometry of contact transformations. Geom. Funct. Anal., 10(6):1448-1476, 2000.

[7] Yakov Eliashberg. Topological characterization of Stein manifolds of dimension > 2. Internat. J. Math., 1(1):29-46, 1990.

[8] Yakov Eliashberg, Sang Seon Kim, and Leonid Polterovich. Geometry of contact transformations and domains: orderability versus squeezing. Geom. Topol., 10:1635-1747, 2006.

[9] F. Gironella. On some examples and constructions of contact manifolds. ArXiv e-prints, November 2017.

[10] Larry Guth. Symplectic embeddings of polydisks. Invent. Math., 172(3):477-489, 2008.

[11] Yang Huang. On plastikstufe, bordered Legendrian open book and overtwisted contact structures. $J$. Topol., 10(3): 720-743, 2017.

[12] Klaus Niederkrüger. The plastikstufe - a generalization of the overtwisted disk to higher dimensions. Algebr. Geom. Topol., 6:2473-2508, 2006.

[13] Klaus Niederkrüger and Francisco Presas. Some remarks on the size of tubular neighborhoods in contact topology and fillability. Geom. Topol., 14(2):719-754, 2010.

[14] Francisco Presas et al. A class of non-fillable contact structures. Geom. Topol., 11(4): 2203-2225, 2007.

[15] Sheila Sandon. Floer homology for translated points. 2016.

Instituto de Ciencias Matematicas CSiC-UAM-UCM-UC3M, C. Nicolás Cabrera, 13-15, 28049, MADRID, SPAIN

E-mail address: luishcorbato@mat.ucm.es

Departamento de Álgebra, Geometría y Topología, Universidad Complutense de Madrid, Plaza De Ciencias 3, 28040 Madrid, Spain

E-mail address: Immerchan@ucm.es

Instituto de Ciencias Matematicas CSiC-UAM-UCM-UC3M, C. Nicolás Cabrera, 13-15, 28049, MADRID, SPAIN

E-mail address: fpresas@icmat.es 